PROCEEDINGS OF THE

AMERICAN MATHEMATICAL SOCIETY

Volume 128, Number 1, Pages 65-74

S 0002-9939(99)05128-X

Article electronically published on June 30, 1999

\title{
REARRANGEMENT OF HARDY-LITTLEWOOD MAXIMAL FUNCTIONS IN LORENTZ SPACES
}

\author{
JESÚS BASTERO, MARIO MILMAN, AND FRANCISCO J. RUIZ \\ (Communicated by Frederick W. Gehring)
}

\begin{abstract}
For the classical Hardy-Littlewood maximal function $M f$, a well known and important estimate due to Herz and Stein gives the equivalence $(M f)^{*}(t) \sim f^{* *}(t)$. In the present note, we study the validity of analogous estimates for maximal operators of the form

$$
M_{p, q} f(x)=\sup _{x \in Q} \frac{\left\|f \chi_{Q}\right\|_{p, q}}{\left\|\chi_{Q}\right\|_{p, q}},
$$

where $\|\cdot\|_{p, q}$ denotes the Lorentz space $L(p, q)$-norm.
\end{abstract}

\section{INTRODUCTION}

The Hardy-Littlewood maximal function $M$ plays a central role in classical harmonic analysis, differentiation theory and PDE's. It is well known that the maximal operator $M$ is of weak type $(1,1)$ and strong type $(\infty, \infty)$ from where it follows readily, for example using K-functionals (see [BS]), that there exists an absolute constant $C>0$ such that

$$
(M f)^{*}(t) \leq C f^{* *}(t), \forall t>0, \forall f \in L_{l o c}^{1}\left(\mathbb{R}^{n}\right),
$$

where $^{*}$ denotes non-increasing rearrangement, and $f^{* *}(t)=\frac{1}{t} \int_{0}^{t} f^{*}(s) d s$. is,

Herz (cf. $[\mathrm{H}]$, and also [BS]) proved that the reverse inequality is also true, that

$$
f^{* *}(t) \leq c(M f)^{*}(t), t>0 .
$$

Inequalities (1) and (2) contain the basic information to study $M$, and the operators it controls, in rearrangement invariant function spaces. We refer to [AKMP] for a recent and exhaustive study of inequalities (1) and (2) where the underlying measure is more general than Lebesgue measure.

Recall that the maximal operator $M$ is defined by

$$
M f(x)=\sup _{x \in Q} \frac{1}{|Q|} \int_{Q}|f(t)| d t=\sup _{x \in Q} \frac{\left\|f \chi_{Q}\right\|_{L^{1}}}{\left\|\chi_{Q}\right\|_{L^{1}}} .
$$

Received by the editors March 2, 1998.

1991 Mathematics Subject Classification. Primary 42B25, 46E30.

Key words and phrases. Maximal functions, rearrangement inequalities, Lorentz spaces.

The first author was partially supported by DGICYT PB94-1185.

The third author was partially supported by DGICYT and IER. 
A commonly used variant of the maximal operator, $M_{p} f=\left(M|f|^{p}\right)^{1 / p}$, is obtained by means of replacing $L^{1}$ averages with $L^{p}$-averages. More generally Stein $[\mathrm{S}]$, in order to obtain certain end point results in differentiation theory, introduced maximal operators associated with $L(p, q)$ averages as follows. Let $1 \leq p<+\infty$, $1 \leq q \leq+\infty$; then Stein defines

$$
M_{p, q} f(x)=\sup _{x \in Q} \frac{\left\|f \chi_{Q}\right\|_{p, q}}{\left\|\chi_{Q}\right\|_{p, q}}=\sup _{x \in Q} \frac{\left\|f \chi_{Q}\right\|_{p, q}}{|Q|^{1 / p}} .
$$

These operators have been also considered by other authors, for instance see $[\mathrm{N}]$, $[\mathrm{LN}]$ and $[\mathrm{P}]$.

It is a basic fact of real interpolation (see $[\mathrm{BS}]$ ) that $f^{* *}(t)$ can be obtained in terms of the $K$-functional for the pair $\left(L^{1}, L^{\infty}\right)$ as

$$
f^{* *}(t)=\frac{K\left(t, f ; L^{1}, L^{\infty}\right)}{t},
$$

where for a compatible pair of Banach spaces $(X, Y), f \in X+Y, t>0$,

$$
K(t, f ; X, Y)=\inf \left\{\|x\|_{X}+t\|y\|_{Y}\right\},
$$

and the inf runs over all possible decompositions $f=x+y$ with $x \in X, y \in Y$.

From the definition of $M_{p}$ given above it follows readily, using (1), (2) and the reiteration theorem, that

$$
\left(M_{p} f\right)^{*}(t) \approx t^{-1 / p} K\left(t^{1 / p}, f ; L^{p}, L^{\infty}\right) .
$$

Therefore one is led to ask if a similar relationship exists between $\left(M_{p, q} f\right)^{*}(t)$ and the corresponding $K$-functional for the pair $\left(L(p, q), L^{\infty}\right)$, which is given by

$$
\frac{K\left(t^{1 / p}, f ; L(p, q), L^{\infty}\right)}{t^{1 / p}} \approx \frac{1}{t^{1 / p}}\left(\int_{0}^{t} f^{*}(s)^{q} s^{q / p-1} d s\right)^{1 / q}=\frac{c}{t^{1 / p}}\left\|f^{*} \chi_{(0, t)}\right\|_{p, q} .
$$

As we shall show below the somewhat surprising answer to this question is: no! The two cases we need to consider $p<q$ and $q<p$ turn out to be very different from each other. In fact for $q<p$, the $L(p, q)$ version of inequality (1) is known to hold as can be readily seen since $M_{p, q}$ is bounded from $L(p, q)$ into $L(p, \infty)$ and from $L^{\infty}$ into $L^{\infty}$ (cf. [S] and also [LN] for a different proof). For $p<q$, the validity of the corresponding $L(p, q)$ version of inequality (1) must be ruled out since, as is well known, $M_{p, q}$ is not bounded from $L(p, q)$ into $L(p, \infty)$ (cf. [S]).

Our purpose in this note is to complete these results by showing in section 2 that the $L(p, q)$ version of inequality (2) is true when $q>p$ and false when $q<p$. In view of these negative results it is natural to ask: what is the appropriate maximal operator associated with the $K$-functional for the pair $\left(L(p, q), L^{\infty}\right)$ so that the corresponding version of Herz's theorem holds? In section 3 we provide an answer by means of finding an improvement on the operator $\left(M_{p, q} f\right)^{*}$.

It will be convenient for us to work in the more general context of r.i. spaces. Indeed the added generality does not complicate the proofs and helps one to see better how the geometrical properties of the $L(p, q)$ spaces intervene in the analysis of the cases $q>p$ or $p>q$.

As usual, a Banach space $\left(X,\|\cdot\|_{X}\right)$ of real-valued, locally integrable, Lebesgue measurable functions on $\mathbb{R}^{n}$ is said to be a r.i. space if it satisfies the following conditions: 
i) If $g^{*} \leq f^{*}$ and $f \in X$, then $g \in X$ with $\|g\|_{X} \leq\|f\|_{X}$ ( $f^{*}$ denotes the non-increasing rearrangement of the function $f$ ).

ii) If $A$ is a Lebesgue measurable set of finite measure, then $\chi_{A} \in X$.

iii) $0 \leq f_{n} \uparrow, \sup _{n \in \mathbb{N}}\left\|f_{n}\right\|_{X} \leq M$, imply that $f=\sup f_{n} \in X$ and $\|f\|_{X}=$ $\sup _{n \in \mathbb{N}}\left\|f_{n}\right\|_{X}$.

For each r.i. space $X$ on $\mathbb{R}^{n}$, a r.i. space $\bar{X}$ on $I=(0,+\infty)$ is associated such that $f \in X$ if and only if $f^{*} \in \bar{X}$ and $\|f\|_{X}=\left\|f^{*}\right\|_{\bar{X}}$ (see [BS]).

The fundamental function of a r.i. Banach space $X$ is defined by

$$
\Phi(t)=\Phi_{X}(t)=\left\|\chi_{[0, t)}\right\|_{\bar{X}}, t>0 .
$$

We will denote by $M^{*}(X)$ the space of all measurable functions for which

$$
\|f\|_{M^{*}(X)}=\sup _{t \in I} \Phi_{X}(t) f^{*}(t)<\infty .
$$

The function $\|\cdot\|_{M^{*}(X)}$ is a quasinorm on $M^{*}(X)$.

For any measurable function $f$ such that $f \chi_{Q} \in X$, we define the maximal operator

$$
M_{X} f(x)=\sup _{x \in Q} \frac{\left\|f \chi_{Q}\right\|_{X}}{\left\|\chi_{Q}\right\|_{X}}
$$

where the supremum is taken over all cubes $Q \subset \mathbb{R}^{n}$ which contain $x$ with sides parallel to the coordinate axes.

Since a conditional expectation operator is a norm one projection in any r.i. space, it is clear that for any cube $Q$ we have

$$
\left\|\left(\frac{1}{|Q|} \int_{Q} f\right) \chi_{Q}\right\|_{X} \leq\left\|f \chi_{Q}\right\|_{X}
$$

and therefore, $M f \leq M_{X} f$, where as usual $M f$ is the classical Hardy-Littlewood maximal function.

Definition. Let $X$ be a r.i. space and let $\phi:[0,+\infty) \longrightarrow[0,+\infty)$ an increasing bijection. $X$ is said to satisfy an upper $\phi$-estimate (resp. lower $\phi$-estimate) if there exists a constant $M<+\infty$ such that, for every choice $\left\{f_{i}\right\}_{i=1}^{n}$ of functions in $X$ with disjoint supports,

$$
\left\|\sum_{i=1}^{n} f_{i}\right\|_{X} \leq M \phi\left(\sum_{i=1}^{n} \phi^{-1}\left(\left\|f_{i}\right\|_{X}\right)\right),
$$

respectively

$$
\left\|\sum_{i=1}^{n} f_{i}\right\|_{X} \geq M \phi\left(\sum_{i=1}^{n} \phi^{-1}\left(\left\|f_{i}\right\|_{X}\right)\right) .
$$

In the special case when $\phi(t)=t^{1 / p}$ we recover the well known notions of lower and upper p-estimates (see [LT]).

Theorem 1. Let $X$ be a r.i. space with fundamental function $\Phi$. If $X$ satisfies a lower $\Phi$-estimate, then $M_{X}: X \longrightarrow M^{*}(X)$ is a bounded operator. In other words, there exists $C>0$ such that for all $f \in X$ we have

$$
\sup _{t} \Phi(t)\left(M_{X} f\right)^{*}(t) \leq C\|f\|_{X}
$$


As a consequence,

$$
\left(M_{X} f\right)^{*}(t) \leq \frac{C}{\Phi(t)}\left\|f^{*} \chi_{(0, t)}\right\|_{X}, \quad \forall t>0 .
$$

Proof. Let $f \in X$, in terms distribution functions we have to prove that

$$
\Phi\left(\left|\left\{x: M_{X} f(x)>\lambda\right\}\right|\right) \leq \frac{C}{\lambda}\|f\|_{X}, \lambda>0 .
$$

Let $\Omega=\left\{x: M_{X} f(x)>\lambda\right\}$. Using the definition of $M_{X}$ and a standard covering lemma (cf. [BS], pg. 118), it is possible to choose a countable family $\mathbf{F}$ of cubes $\{Q\}_{i \in J}$ with pairwise disjoint interiors and such that

$$
|\Omega| \leq 4^{n} \sum_{i \in J}\left|Q_{i}\right|, \quad \frac{\left\|f \chi_{Q_{i}}\right\|_{X}}{\left\|\chi_{Q_{i}}\right\|_{X}}>\lambda, \forall i \in J
$$

Moreover, if $Q \in \mathbf{F}$, then

$$
\Phi(|Q|)<\left\|\left(\frac{f}{\lambda}\right) \chi_{Q}\right\|_{X}, \quad|Q|<\Phi^{-1}\left(\left\|\left(\frac{f}{\lambda}\right) \chi_{Q}\right\|_{X}\right) .
$$

Therefore, using that $\Phi(5 t) \leq 5 \Phi(t)$ and the lower $\Phi$-estimate, we get

$$
\Phi(|\Omega|) \leq 5 \Phi\left(\sum_{i \in J} \Phi^{-1}\left(\left\|\left(\frac{f}{\lambda}\right) \chi_{Q_{i}}\right\|_{X}\right)\right) \leq 5 M\left\|\sum_{i \in J}\left(\frac{f}{\lambda}\right) \chi_{Q_{i}}\right\|_{X} \leq \frac{5 M}{\lambda}\|f\|_{X} .
$$

This proves the first part of the theorem. The proof of the second part is a routine argument in interpolation theory. Indeed, since $M_{X}$ is a bounded operator on $L^{\infty}$, we have that, $\forall t>0$,

$$
K\left(\Phi(t), M_{X} f, M^{*}(X), L^{\infty}\right) \leq C K\left(\Phi(t), f, X, L^{\infty}\right) .
$$

Now, we recall that the left-hand side of this inequality is equivalent to

$$
\sup _{s \leq t}\left(M_{X} f\right)^{*}(s) \Phi(s)
$$

while the right-hand side is equivalent to

$$
\left\|f^{*} \chi_{(0, t)}\right\|_{X}
$$

(see $[\mathrm{BR}])$, and the result follows.

Theorem 2. Let $X$ be a r.i. space with fundamental function $\Phi$. If $X$ satisfies an upper $\Phi$-estimate, then there exists and absolute constant $C>0$ such that $\forall f \in X, t>0$ we have

$$
\left(M_{X} f\right)^{*}(t) \geq \frac{C}{\Phi(t)}\left\|f^{*} \chi_{(0, t)}\right\|_{X} .
$$

Proof. Fix $f \in X, t>0$. Let $\alpha=\left(M_{X} f\right)^{*}(t)$ and $\Omega=\left\{x: M_{X} f(x)>\alpha\right\}$. Following [BS], pgs. 122-123, we can choose a sequence of dyadic cubes $\left\{Q_{i}\right\}_{i \in J}$ with pairwise disjoint interiors, which covers $\Omega$, and such that

$$
\sum_{i \in J}\left|Q_{i}\right| \leq C|\Omega| \leq C t, \quad \frac{\left\|f \chi_{Q_{i}}\right\|_{X}}{\left\|\chi_{Q_{i}}\right\|_{X}} \leq \alpha, \forall i \in J .
$$

Then, we decompose

$$
f=\sum_{i \in J} f \chi_{Q_{i}}+h=g+h .
$$


Using the upper $\Phi$-estimate, we get

$$
\begin{aligned}
& \left\|\frac{g}{\alpha}\right\|_{X} \leq M \Phi\left(\sum_{i \in J} \Phi^{-1}\left(\left\|\frac{f}{\alpha} \chi_{Q_{i}}\right\|_{X}\right)\right) \\
& \leq M \Phi\left(\sum_{i \in J} \Phi^{-1}\left(\Phi\left(\left|Q_{i}\right|\right)\right)\right) \leq C \Phi(t) .
\end{aligned}
$$

Thus,

$$
\|g\|_{X} \leq C\left(M_{X} f\right)^{*}(t) \Phi(t) .
$$

On the other hand, since $f \leq M f \leq M_{X} f$ a.e., we have

$$
\|h\|_{\infty} \leq\left(M_{X} f\right)^{*}(t) .
$$

Then using $[\mathrm{BR}]$ and the definition of the $K$-functional we obtain

$$
\frac{1}{\Phi(t)}\left\|f^{*} \chi_{(0, t)}\right\|_{X}=\frac{1}{\Phi(t)} K\left(\Phi(t), f, X, L^{\infty}\right) \leq C\left(M_{X} f\right)^{*}(t) .
$$

We now turn to study the case when $X=L(p, q)$ is a Lorentz space; in this case the fundamental function of $X$ is $\Phi(t)=t^{1 / p}$. We shall need the following

Lemma. Let $p \in(1,+\infty)$, then

i) If $1<p \leq q \leq+\infty$, then $L(p, q)$ satisfies an upper $p$-estimate.

ii) If $1 \leq q \leq p, L(p, q)$ satisfies a lower $p$-estimate.

Proof. Recall that, in terms of the distribution function, an expression equivalent to the $L(p, q)$-norm can be given as follows:

$$
\|f\|_{p, q}=\left(\int_{0}^{\infty}\left(\lambda_{f}(s)\right)^{q / p} s^{q-1} d s\right)^{1 / q}
$$

with the usual modification when $q=+\infty$. Given a sum $f=\sum f_{i}$ where the $f_{i}$ have disjoint supports, it is clear that

$$
\lambda_{f}(s)=\sum \lambda_{f_{i}}(s)
$$

Therefore in the case $q<\infty$ the corresponding lower and upper p-estimates concern only the interchange of sums and integrals and can be obtained easily using Minkowski's vector-valued inequality, while the case $q=+\infty$ is even simpler.

Corollary. Let $p \in(1,+\infty)$. Then:

i) If $1<p \leq q \leq+\infty$, then there exists a constant $C>0$ such that $\forall f \in X, t>0$,

$$
\left(M_{p, q} f\right)^{*}(t) \geq \frac{C}{t^{1 / p}}\left(\int_{0}^{t} f^{*}(s)^{q} s^{q / p-1} d s\right)^{1 / q}
$$

(with the usual modification if $q=+\infty$ ).

ii) If $1 \leq q \leq p$, then there exists a constant $C>0$ such that $\forall f \in X, t>0$,

$$
\left(M_{p, q} f\right)^{*}(t) \leq \frac{C}{t^{1 / p}}\left(\int_{0}^{t} f^{*}(s)^{q} s^{q / p-1} d s\right)^{1 / q} .
$$


We now focus on the validity of the reverse inequalities which correspond to those stated in the previous corollary. We remark that, as we mentioned in the introduction, under the conditions of $\mathbf{i}$ ), the corresponding reverse inequality cannot be true. Our next theorem completes this result by showing that the reverse inequality in ii) is not true either. Since there is no loss of generality, we shall work in dimension one.

Theorem 3. There exists a function $f$ defined on $\mathbb{R}$ for which $\int_{0}^{t} f^{*}(x) x^{1 / p-1} d x=$ $\infty$ for all $t>0$ while $\left(M_{p, 1} f\right)^{*}(t)<\infty$, for all $t>0$.

Proof. Let

$$
h(x)=\sum_{k=1}^{\infty} \frac{2^{k / p}}{k} \chi_{\left[2^{-k}, 2^{-k+1}\right)}(x), x>0 .
$$

Since

$$
h(x) \geq C \frac{x^{-1 / p}}{|\log x|}, 0<x<1 / 2
$$

it is clear that

$$
\int_{0}^{t} h(x) x^{1 / p-1} d x=+\infty, \forall t>0 .
$$

Now, we want to distribute the values of $h(x)$ in a convenient form. Let $A_{1}=$ $\left[a_{1}, b_{1}\right]=\left[0,2^{-1}\right], A_{2}=\left[a_{2}, b_{2}\right]=\left[b_{1}+2^{2}, b_{1}+2^{2}+2^{-2}\right]$ and, in general, $A_{k}=$ $\left[a_{k}, b_{k}\right]=\left[b_{k-1}+2^{k}, b_{k-1}+2^{k}+2^{-k}\right], k \in \mathbb{N}$. Let

$$
f=\sum_{k=1}^{\infty} \frac{2^{k / p}}{k} \chi_{A_{k}} .
$$

It is clear that the rearrangement of $f$ is the function $h$. In order to estimate its maximal function, we need the following facts which have straightforward proofs (in what follows $Q$ stands for an interval in $\mathbb{R}$ ):

i) If $Q \cap A_{i} \neq \emptyset$ for $i=k, k+1, \ldots l, l>k$, then

$$
\frac{\left\|f \chi_{Q}\right\|_{p, 1}}{|Q|^{1 / p}} \leq \frac{2^{l / p}}{l} \frac{\left\|\chi_{A_{k} \cup \cdots \cup A_{l}}\right\|_{p, 1}}{2^{l / p}} \leq \frac{1}{l} .
$$

ii) Let $b_{k}<x<a_{k+1}$. If $Q=[z, x]$ with $z \in A_{k}$, then

$$
\frac{\left\|f \chi_{Q}\right\|_{p, 1}}{|Q|^{1 / p}} \leq \frac{1}{k\left|x-a_{k}\right|^{1 / p}} .
$$

iii) Let $b_{k}<x<a_{k+1}$. If $Q=[x, y]$ with $y \in A_{k+1}$, then

$$
\frac{\left\|f \chi_{Q}\right\|_{p, 1}}{|Q|^{1 / p}} \leq \frac{1}{(k+1)\left|b_{k+1}-x\right|^{1 / p}} .
$$

Now, given $s>0$, we choose $k_{0}$ such that $s k_{0}>1$. Let $k \geq k_{0}$. If

$$
b_{k}+\frac{1}{k^{p} s^{p}} \leq x \leq a_{k+1}-\frac{1}{(k+1)^{p} s^{p}},
$$


then $M f(x) \leq s$. On the other hand, if we take $y_{0}<0$ such that

$$
\max _{1 \leq k \leq k_{0}} \frac{s^{j / p}}{k\left(\left|y_{0}\right|+2+\cdots+2^{k}\right)^{1 / p}}<s
$$

then

$$
\begin{aligned}
& \{x \in \mathbb{R} ; M f(x)>s\} \\
& \quad \subseteq\left(y_{0}, b_{k_{0}}\right] \bigcup_{k \geq k_{0}}\left(A_{k} \cup\left(b_{k}, b_{k}+\frac{1}{k^{p} s^{p}}\right) \cup\left(a_{k+1}-\frac{1}{(k+1)^{p} s^{p}}, a_{k+1}\right)\right),
\end{aligned}
$$

and we obtain the estimate

$$
\lambda_{M_{p, 1} f}(s) \leq\left|b_{k_{0}}-y_{0}\right|+\sum_{k_{0}+1}^{\infty}\left(\frac{1}{2^{k}}+\frac{1}{s^{p} k^{p}}+\frac{1}{s^{p}(k+1)^{p}}\right)<\infty .
$$

Moreover, $\lim _{s \rightarrow \infty} \lambda_{M_{p, 1} f}(s)=0$, and therefore

$$
\left(M_{p, 1} f\right)^{*}(t)<\infty, \forall t>0 .
$$

Remark. The counterexample given above can be easily extended to the case $1 \leq$ $q<p$ since we have

$$
M_{p, q} f(x)=\left(M_{r, 1}\left(f^{q}\right)\right)^{1 / q}, \quad r=p / q .
$$

We recall that a mapping $\tau$ from $\mathbb{R}^{n}$ into $\mathbb{R}^{n}$ is said to be a measure-preserving transformation if, whenever $A$ is a Borel subset of $\mathbb{R}^{n}$, the set $\tau^{-1}(A)$ is also Borel set, and $\left|\tau^{-1}(A)\right|=|A|$.

Theorem 4. Let $X$ be a r.i. space. Then there exists an absolute constant $C>0$ such that, for any function $f \in X+L^{\infty}$,

$$
c \inf _{\tau}\left(M_{X}(f \circ \tau)\right)^{*}(t) \leq \frac{1}{\phi(t)}\left\|f^{*} \chi_{[0, t]}\right\|_{X} \leq C \sup _{\tau}\left(M_{X}(f \circ \tau)\right)^{*}(t)
$$

for all $t>0$, where $\tau$ runs through all measure-preserving transformations.

Proof. We begin by proving the second inequality. Fix $t$ and $f$. We can suppose that $\alpha=\frac{1}{\Phi(t)}\left\|f^{*} \chi_{[0, t]}\right\|_{X}>0$. Without loss of generality, we can also suppose that $f$ is Borel measurable. Then there exists a Borel set $B$ in $\mathbb{R}^{n}$ such that $|B|=t$ and satisfying

$$
\left\{x \in \mathbb{R}^{n} ;|f(x)|>f^{*}(t)\right\} \subseteq B \subseteq\left\{x \in \mathbb{R}^{n} ;|f(x)| \geq f^{*}(t)\right\} .
$$

Now, given a cube $Q_{0}$ with measure $t$ it is always possible to find a bijective measurepreserving transformation $\tau$ such that $\tau\left(Q_{0}\right)=B$ (except perhaps null measurable subsets of $Q$ and $B$ ). (This statement can be readily proved using [R], p. 315, Theorem 15.2, Theorem 15.13; see also [W] for the one dimensional case.) That is, we can find a measure-preserving transformation $\tau$ (which, of course, depends on $f$ and $t$ ) such that

$$
(f \circ \tau) \chi_{Q_{0}}=f \chi_{B} \quad \text { a.e. }
$$


Then

$$
\frac{\left\|(f \circ \tau) \chi_{Q_{0}}\right\|_{X}}{\left\|\chi_{Q_{0}}\right\|_{X}}=\frac{\left\|f \chi_{B}\right\|_{X}}{\Phi(t)}=\frac{\left\|f^{*} \chi_{[0, t]}\right\|_{X}}{\Phi(t)} .
$$

If we take $Q_{1}=2 Q_{0}$, the cube with equal center and double the side of $Q_{0}$, then since $\Phi\left(\left|Q_{1}\right|\right) \leq 2^{n} \Phi(t)$, we have

$$
\frac{\left\|(f \circ \tau) \chi_{Q_{1}}\right\|_{X}}{\left\|\chi_{Q_{1}}\right\|_{X}} \geq \frac{\alpha}{2^{n}}>\frac{\alpha}{2.2^{n}} .
$$

Therefore, $M_{X}(f \circ \tau)(x)>\frac{\alpha}{2.2^{n}}$ for all $x \in Q_{1}$ and

$$
\left|\left\{s \in(0, \infty) ;\left(M_{x}(f \circ \tau)\right)^{*}(s)>\frac{\alpha}{4.2^{n}}\right\}\right| \geq\left|Q_{1}\right|=2^{n}\left|Q_{0}\right|>t .
$$

Thus,

$$
\left(M_{X}(f \circ \tau)\right)^{*}(t)>\frac{\alpha}{4.2^{n}},
$$

and the second inequality is proved.

It remains to prove the first inequality. Let $t>0$ and suppose, as we may, that $\alpha=\frac{1}{\Phi(t)}\left\|f^{*} \chi_{[0, t]}\right\|_{X}>0($ since otherwise $f=0)$. By repeating the arguments as before we find the same $Q_{0}, Q_{1}$ and $\tau$.

We are going to estimate $M_{X}(f \circ \tau)(x)$ for $x \notin Q_{1}$. Let $Q$ be a cube such that $x \in Q$. If $Q \cap Q_{0}=\emptyset$, then

$$
\frac{\left\|(f \circ \tau) \chi_{Q}\right\|_{X}}{\left\|\chi_{Q}\right\|_{X}} \leq f^{*}(t) \leq \frac{\left\|f^{*} \chi_{[0, t]}\right\|_{X}}{\Phi(t)}=\alpha .
$$

If $Q \cap Q_{0} \neq \emptyset$, then there exists a cube $\bar{Q} \subseteq Q \cap Q_{1}$ such that $\left|Q_{0}\right|=|\bar{Q}|$. Thus

$$
\begin{aligned}
\frac{\left\|(f \circ \tau) \chi_{Q}\right\|_{X}}{\left\|\chi_{Q}\right\|_{X}} & \leq \frac{\left\|(f \circ \tau) \chi_{Q \backslash Q_{0}}\right\|_{X}}{\left\|\chi_{Q}\right\|_{X}}+\frac{\left\|(f \circ \tau) \chi_{Q \cap Q_{0}}\right\|_{X}}{\left\|\chi_{Q}\right\|_{X}} \\
& \leq f^{*}(t) \frac{\left\|\chi_{Q \backslash Q_{0}}\right\|_{X}}{\left\|\chi_{Q}\right\|_{X}}+\frac{\left\|(f \circ \tau) \chi_{Q_{0}}\right\|_{X}}{\left\|\chi_{Q_{0}}\right\|_{X}} \\
& \leq f^{*}(t)+\alpha \leq 2 \alpha .
\end{aligned}
$$

Therefore,

$$
\left|\left\{x \in \mathbb{R}^{n} ; M(f \circ \tau)(x)>3 \alpha\right\}\right| \leq\left|Q_{1}\right|=2^{n} t,
$$

and so

$$
(M(f \circ \tau))^{*}\left(2^{n} t\right) \leq 3 \alpha .
$$

In particular, if we apply the preceding argument to $t_{0}=t / 2^{n}<t$, we find $\tau_{0}$ for which

$$
\begin{aligned}
\left(M\left(f \circ \tau_{0}\right)\right)^{*}(t) & =\left(M\left(f \circ \tau_{0}\right)\right)^{*}\left(2^{n} t_{0}\right) \leq 3 \frac{1}{\Phi\left(t_{0}\right)}\left\|f^{*} \chi_{\left[0, t_{0}\right]}\right\|_{X} \\
& \leq 3 \frac{2^{n}}{\Phi(t)}\left\|f^{*} \chi_{[0, t]}\right\|_{X}=3.2^{n} \alpha,
\end{aligned}
$$

and the desired inequality follows by taking infimum. 
Corollary 2. Let $p \in(1,+\infty)$. Then:

i) If $1 \leq q \leq p$,

$$
\sup _{\tau}\left(M_{p, q}(f \circ \tau)\right)^{*}(t) \sim \frac{1}{t^{1 / p}}\left(\int_{0}^{t} f^{*}(s)^{q} s^{q / p-1} d s\right)^{1 / q} .
$$

ii) If $1<p \leq q \leq \infty$,

$$
\inf _{\tau}\left(M_{p, q}(f \circ \tau)\right)^{*}(t) \sim \frac{1}{t^{1 / p}}\left(\int_{0}^{t} f^{*}(s)^{q} s^{q / p-1} d s\right)^{1 / q} .
$$

Proof. i) One inequality follows directly from the corollary to Theorem 2. To prove the reverse inequality we note that since $f$ and $f \circ \tau$ have the same distribution, $M_{X}(f \circ \tau)$ is a bounded operator from $X$ into $M^{*}(X)$ and from $L^{\infty}$ into $L^{\infty}$. Therefore (see proof of Theorem 1)

$$
\left(M_{p, q}(f \circ \tau)\right)^{*}(t) \leq C \frac{1}{t^{1 / p}}\left(\int_{0}^{t} f^{*}(s)^{q} s^{q / p-1} d s\right)^{1 / q},
$$

and the result follows taking the supremum over all $\tau$.

ii) As in the previous proof, one inequality comes again from Theorem 2, while the other follows from

$$
\left(M_{p, q}(f \circ \tau)\right)^{*}(t) \geq C \frac{1}{t^{1 / p}}\left(\int_{0}^{t}(f \circ \tau)^{*}(s)^{q} s^{q / p-1} d s\right)^{1 / q}
$$

where the constant $C$ is independent of $\tau$ (see proof of Theorem 2).

\section{ACKNOWLEDGEMENT}

We are grateful to Dan Waterman and Togo Nishiura for their help with a question on measure preserving transformations that arose in the proof of Theorem 4.

\section{REFERENCES}

[AKMP] I.U. Asekritova, N.Y. Krugljak, L. Maligranda and L.E. Persson, Distribution and rearrangement estimates of the maximal function and interpolation, Studia Math. 124 (2) (1997), 107-132. MR 98g:46032

[BR] J. Bastero and F. Ruiz, Elementary reverse Holder type inequalities with application to operator interpolation theory, Proc. A.M.S. 124 (10) (1996), 3183-3192. MR 96m:46042

[BS] C. Bennett and R. Sharpley, Interpolation of operators, Academic Press, 1988. MR 89e: 46001

$[\mathrm{H}] \quad$ C. Herz, The Hardy-Littlewood maximal theorem, Symposium on Harmonic Analysis, University of Warwick, 1968.

[LN] M.A. Leckband and C.J. Neugebauer, Weighted iterates and variants of the HardyLittlewood maximal operator, Trans. A.M.S. 275 (1) (1983), 51-61. MR 85c:42021

[LT] J. Lindenstrauss and L. Tzafriri, Classical Banach Spaces II, Springer Verlag, 1979. MR 81c:46001

[N] C.J. Neugebauer, Iterations of Hardy-Littlewood maximal functions, Proc. A.M.S. 101 (1) (3) (1987), 272-276. MR 88k:42014

[P] C. Pérez, Endpoint estimates for commutators of singular integral operators, Journal of Func. Anal. 128 (1) (1995), 163-185. MR 95j:42011

[R] H.L. Royden, Real Analysis. 2nd. edition, MacMillan Publishing Co., Inc., New York, 1968. 
[S] E.M. Stein, Editor's Note: The differentiability of functions in $\mathbb{R}^{n}$, Ann. of Math. 133 (1981), 383-385. MR 84j:35077

[W] D. Waterman, On systems of functions resembling the Walsh system, Michigan Math. J. 29 (1982), 83-87. MR 85a:42039

Department of Mathematics, University of Zaragoza, 50009-Zaragoza, Spain

E-mail address: bastero@posta.unizar.es

Department of Mathematics, Florida Atlantic University, Boca Raton, Florida 33431

E-mail address: milman@acc.fau.edu

Department of Mathematics, University of Zaragoza, 50009-Zaragoza, Spain

E-mail address: fjruiz@posta.unizar.es 